\title{
KFLOW Results of Airloads on HART-II Rotor Blades with Prescribed Blade Deformation
}

\author{
Jeong Hwan Sa*, Jee Woong Kim*, Soo Hyung Park**, Jae Sang Park**, \\ Sung Nam Jung** and Yung Hoon Yu** \\ Department of Aerospace Information Engineering, \\ Konkuk University, Seoul, Korea
}

\begin{abstract}
A three-dimensional compressible Navier-Stokes solver, KFLOW, using overlapped grids has recently been developed to simulate unsteady flow phenomena over helicopter rotor blades. The blade-vortex interaction is predicted for a descending flight using measured blade deformation data. The effects of computational grid resolution and azimuth angle increments on airloads were examined, and computed airloads and vortex trajectories were compared with HART-II wind tunnel data. The current method predicts the BVI phenomena of blade airloads reasonably well. It is found from the present study that a peculiar distribution of vorticity of tip vortices in an approximate azimuth angle range of 90 to 180 degrees can be explained by physics of the shear-layer interaction as well as the dissipation of numerical schemes.
\end{abstract}

Key Words : Computational Fluid Dynamics, HART II rotor, Aeroelastic Simulation, Blade Vortex Interaction

\section{Introduction}

The flowfield around a helicopter is characterized by the constantly changing aerodynamic environment and loads due to the variation with Mach number and Reynolds number along the blade span, interactions between the rotating blades and their wake systems, and aero-elastic effects. One of the most important features over the flowfield around rotor blades is the blade-vortex interaction (BVI), which generates the most annoying and intrusive noise from helicopter rotors [1]. During low speed descending and maneuvering flight, this phenomenon becomes dominant, since the rotor wake remains near the rotor disk plane. It is important to predict the rotor wake accurately to improve the prediction capability for BVI noise and airloads in analysis of the rotorcraft Computational Fluid Dynamics (CFD). However, precise prediction and understanding of the rotor wake are challenging problems, because computed wakes diffuse too rapidly due to grid and numerical dissipation.

In order to improve the understanding and prediction capability of rotor wake aerodynamics, an extensive range of wind tunnel tests and CFD analyses have been performed for descending flights. In 1994, a higher-harmonic Aeroacoustic Rotor Test (HART I) [1] for a 40\% Mach-scaled model rotor of a BO-105 main rotor was conducted by researchers from German DLR, French ONERA, NASA Langley, Dutch DNW and the US AFDD in the open-jet anechoic test section of the German-Dutch Wind tunnel (DNW). A HART II test [2,3] was conducted in 2001 as an extension of the HART I program in order to focus on investigation of the rotor wake and vortex development. With measured data from these tests, a variety of correlation studies have been performed [4-6].

* Graduate Student

** Professor

E-mail : pish@konkuk.ac.kr

Tel : $+82-2-450-4177$

Fax : $+82-2-444-6670$ 
With rapid development of computers and numerical methods, recent studies involving HART I and HART II tests have utilized various CFD solvers based on Euler and Navier-Stokes Equations. For CFD methodologies, progress has been made toward reduction of numerical dissipation causing a diffusion of rotor vortices. Dietz et al. [7] used vortex-adapted Chimera-child grids to reduce the numerical dissipation of the vortex and showed improvement in tip vortex conservation. Brown et al. [8,9] applied the vorticity transport model (VTM) to separate vorticity transport from the numerical dissipation of any Navier-Stokes solver. Higher order spatial discretization schemes and huge numbers of grid points have been exploited in order to prevent rapid dissipation of rotor wakes [10-13]. The computational results reported in these studies generally showed that vortex trajectories and BVI airloads are predicted well with a background-grid spacing smaller than 0.05 chord length. Note that a HART II particle image velocimetry (PIV) experiment shows that the tip vortex has a radius of about 0.05 chord length [3]. For HART II validation, higher order schemes and a grid spacing smaller than 0.05 chord length in the background mesh are recommended to capture rotor vortices, when an overlapping grid system is applied [11].

Visualizations of HART II rotor vortex trailers [5,11,13] show details of the blade-vortex interactions. Dispersion of vortices was explained as a result of the inevitable numerical dissipation. From HART II experiments [2,3] and figures of high resolution $[11,13]$, the tip vortex strengthens at a position just behind the tip as the blade tip passes through the 3rd to 1 st quadrant of the rotor disk, whereas the tip vortex weakens in the 2 nd quadrant. The vortices do not collide with a blade, as seen in the airload distribution over the rotor disk. This is not strongly related to either local tip Mach number or pitch angle, because a strong tip vortex is generated in the 4th quadrant where the effect of the tip Mach number and pitch angle is relatively lower than in other quadrants. This could not be explained by only the numerical dissipation. To the authors' knowledge, no explanation has been offered regarding this phenomenon.

The objective of the present study is to validate the present CFD code that can simulate the aeroelastic problem using the measured blade deformation [14] with the airload data of the HART II baseline (BL) case. Airload prediction capability is assessed through a sensitivity study on grid cell size and time step. Vortex locations are compared with the results of experiments and wake structures are discussed.

\section{Numerical Approach}

\section{KFLOW}

KFLOW [15] is a parallelized multiblock structured, Navier-Stokes solver. It is capable of computing time-accurate moving body problems by using a parallelized CHIMERA overlapping grid system. 2nd-order accurate dual-time stepping [16] combined with the diagonalized alternating - direction implicit (DADI) method [17] is used to advance the solution in time. This allows the use of a large time increment and makes it possible to maintain temporal accuracy. The dual-time stepping also eliminates factorization and linearization errors by iterating the solutions in pseudo-time. The number of sub-iterations at each physical time step is around 5, sufficient for accurate simulations of rotor blades through numerical experiments.

Six turbulence models, including Spalart-Allmaras and SST k- $\omega$ turbulent models, have been implemented [17]. Low Mach-number preconditioning with a multigrid can be applied to low-speed compressible unsteady flows. In this study, the compressible Euler equations are only considered to simulate the present baseline (BL) case around the HART II rotor, since viscous boundary layers are well attached over blades for the present case and lift and moment characteristics are marginally affected by viscous effects [15]. 
In this paper, the inviscid flux is calculated by Roe flux difference splitting and the 5th order Weighted Essentially Non-Oscillatory (WENO) scheme [18], as spatial discretization schemes. Characteristic boundary conditions using the Riemann invariant are applied at the farfield boundary. At the solid wall of the rotor blade, a no-slip or slip condition is applied.

In aeroelastic simulations deformations of the rotor blade bring about changes in grid cell volumes, necessitating rigorous enforcement of the GCL (Geometric Conservation Law) [19], which states that the increase of a moving cell must be equal to the sum of the variations along the surfaces that enclose the volume. The GCL is applied to meet the conservative relations of the surfaces and volumes of the control cells in a moving grid. A prescribed deformation obtained from a HART II experiment is imposed on the blade movement at each time step. The converged solution at each physical time step is updated by using a modified volume calculated from the GCL.

\section{Overlapped Grid System}

The Chimera method offers not only efficient grid generation but the capability to handle relative movement of multiple bodies. However, the information to connect overlapped grids must be re-established whenever a grid is moved. This process of establishing communication is referred to as "grid assembly". The grid assembly is parallelized with static load balancing based on the decomposed grid system of the flow solver. A coarse-grained communication is optimized with a minimized memory allocation and communication load, since the parallel grid assembly accesses the decomposed geometry data within other processors only by message passing in a distributed memory system, such as a PC cluster. A parallel performance assessment of a moving body overset grid application on a PC cluster was achieved [20].

The overlapped grid method is comprised of two major steps, hole cutting and donor cell identification. The zones of interference scheme and a hole-map algorithm are used for the hole cutting procedure. The stencil-walk and gradient searching methods are adapted for the second step [21]. Solid walls are initially used for hole-cutting surfaces. The gathering of surfaces patches in each body is executed by group communication after regenerating a new communicator among the processors related to the same body, because only a closed surface is used to make the hole-map. Interpolation and donor data in the take and send processors, respectively, are connected by sorting with a tag index. Thus, minimized information of interpolation points is communicated between the 'take' and 'send' processors during the parallel donor searching. A detailed description and evaluation of the performance of the parallelization of grid assembly can be found in [20].
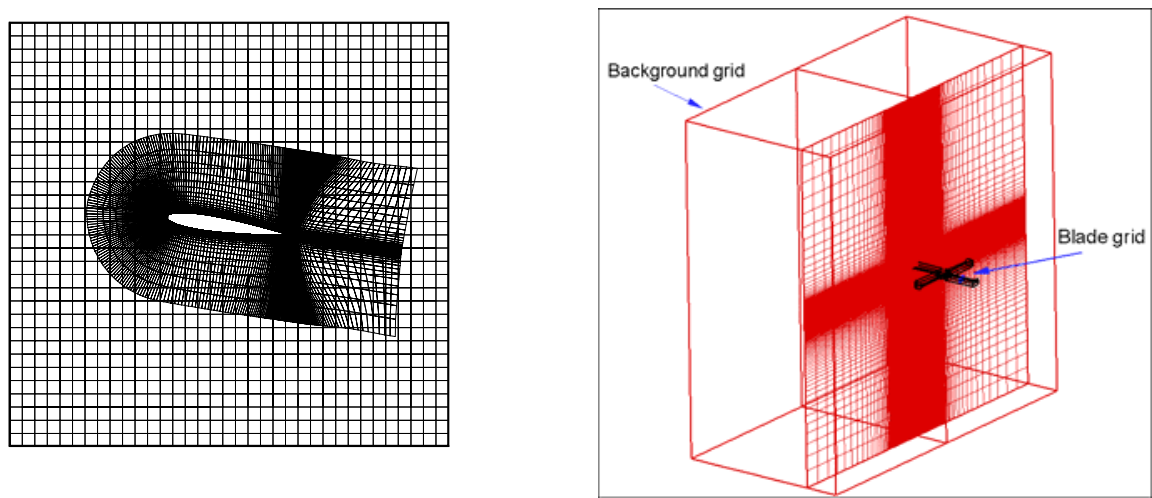

Fig. 1. (Left) close view of C-type blade grid, (Right) perspective view of grid system 
Table 1. Specifications of grid system

\begin{tabular}{ccc}
\hline \hline Grid Type & Coarse & Standard \\
\hline Background & $89 \times 281 \times 281$ & $111 \times 401 \times 401$ \\
\hline $\begin{array}{c}\text { Inner background } \\
\text { spacing }\end{array}$ & $0.15 \mathrm{c}$ & $0.1 \mathrm{c}$ \\
\hline Blade & $169 \times 89 \times 21$ & $169 \times 89 \times 21$ \\
\hline \hline
\end{tabular}

A moving overlapped Chimera grid system with two different types of grids (blade grids, background grids) is used for the present computation. For the HART II rotor blade, $\mathrm{C}-\mathrm{H}$ type grids were generated. Figure 1 shows a close view of the $\mathrm{C}$-type grid around a blade and a perspective view of the grid system for the whole computational domain. The rotor has four blades with a NACA23012 airfoil with a balance tab. The aspect ratio is 16.529 and the twist angle is $-8^{\circ}$. Table 1 shows the specifications for each grid in the case of HART II computation. The blade grids extend 1.5 chord length away from the blade in all directions. To capture the blade-vortex interaction, the body grids are clustered near the leading edges, trailing edges, and blade tip regions. They are also clustered in a normal direction near the wall. The background wake grid has an inner region that extends 8 chord lengths (above), 8 chord lengths (below) from the rotor disk plane, and 2 chord lengths away from the blade tip. This inner region has a uniform spacing in all directions. The farfield boundary is located 5 blade radii from the rotor hub. Two different background grids are generated to check the grid sensitivity. Each size of grid spacing corresponds to $0.15 \mathrm{c}$ and $0.1 \mathrm{c}$, respectively, where $\mathrm{c}$ is the blade chord length.

\section{Results and Discussion}

\section{Problem conditions and prescribed deformation}

HART II experimental data include three representative cases: baseline (BL) case, minimum noise (MN) case, and minimum vibration (MV) case [2,3]. In the present study, computations were performed for the baseline case. The blades rotate only with the first order cyclic pitch control in order to be used as a standard to other conditions. Table 2 shows test conditions such as blade geometry, flow condition, and blade pitch control.

Table 2. Computational condition for BL case

\begin{tabular}{ll}
\hline Rotor radius, $R(\mathrm{~m})$ & 2.0 \\
Blade chord length, $\mathrm{c}(\mathrm{m})$ & 0.121 \\
& $-8.0^{\circ}$ \\
Twist angle $(/ \mathrm{R})$ & $2.5^{\circ}$ \\
Precone angle & $3300 \mathrm{~N}$ \\
Thrust coefficient, $\mathrm{C}_{T}$ & 0.6387 \\
Tip Mach number, $\mathrm{M}_{\mathrm{Tip}}$ & 0.15 \\
Advance ratio & $4.5^{\circ}$ \\
Angle of tip path plane & $20 \mathrm{Nm}$ \\
Roll moment & (advancing side down) \\
& $-20 \mathrm{Nm}$ (nose up) \\
Pitching moment & $3.2 \mathrm{deg}$ \\
\hline Blade motion data $(\mathrm{BL}$ case) & $2.0 \mathrm{deg}$ \\
Collective $\left(\boldsymbol{\theta}_{0}\right)$ & $-1.1 \mathrm{deg}$ \\
Lateral cyclic $\left(\boldsymbol{\theta}_{1 \mathrm{c}}\right)$ & \\
Longitudinal cyclic $\left(\boldsymbol{\theta}_{1 s}\right)$ &
\end{tabular}


For a particular forward speed, the thrust and moment coefficients can be expressed as a function of collective and cyclic pitch. The relationship between the rotor aerodynamic parameters and the pitch angle is nonlinear. A Newton-Raphson iterative method is used to trim the rotor. To reduce computing time a quarter of a revolution is used to compute each derivative in a trim cycle. The trim cycle consists of 2 revolutions of the rotor blade: Three quarters for calculation of derivatives and the remaining quarters for solution iteration.

In order to minimize error arising from discrete data for grid velocity, continuous data [14], which are analytically expressed in both radial and azimuthal directions, are used to prescribe the blade deformation. The blade deformation was measured using a non-intrusive optical method, called Stereo Pattern Recognition, in the HART II tests [22,23]. Three kinds of elastic deflection data (flap, lead-lag, torsion) are available from the HART II web site. The flap deflection bends perpendicularly with respect to the blade surface. The lead-lag deflection is forward or backward bending along the blade surface and torsion is twisting around the quarter chord of the blade. The data were recorded at $15^{\circ}$ intervals of the blade azimuth and the spanwise spacing is $\Delta \mathrm{r} / \mathrm{R}=0.045$. The available measured data are only a set of values defined at discrete azimuthal and radial positions.

An analytical expression by van der Wall [14] is used to allow computations of the smooth deflection of the blade at any radial or azimuthal location and to interpolate over the gaps in the data. The analytical approximation is based on the best fit of the lowest pre-computed mode shapes, which are obtained from the finite element method and a Fourier series for each of these mode shapes. The flap deflection is written as:

$$
\begin{gathered}
z(r, \psi)=\sum_{i=1}^{N_{z}} \phi_{z i}(x) q_{z i}(\psi) \\
\phi_{z_{i}}(x)=\sum_{j=0}^{N_{c}} c_{z_{i j}} x^{j} \quad \text { with } x=\frac{r / R-0.075}{1-0.075} \\
q_{z_{i}}(\psi)=\sum_{k=0}^{N_{h}}\left(a_{z_{i k}} \cos k \psi+b_{z_{i k}} \sin k \psi\right)
\end{gathered}
$$

where $\Phi_{\boldsymbol{~}}$ are the non-dimensional flap mode shapes depending only on the nondimensional radial mode coordinate $\mathbf{x}$, and $\boldsymbol{q}_{\boldsymbol{z} i}$ are the generalized coordinates carrying the time-dependence with respect to the azimuth $\psi$ and the magnitude of the deflection. The coordinate $\mathbf{x}$ is zero at $\mathrm{r} / \mathrm{R}=0.075$ and $\mathrm{x}=1$ at the blade tip. For the lag and torsion modes the same formulation is used, but with their own mode shapes and own generalized coordinates. Fig.2. displays the measured and computed blade deflection data from the analytical expression at 0.996R spanwise location.

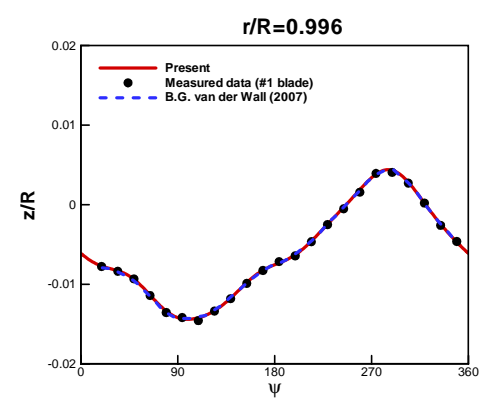

(a) flap

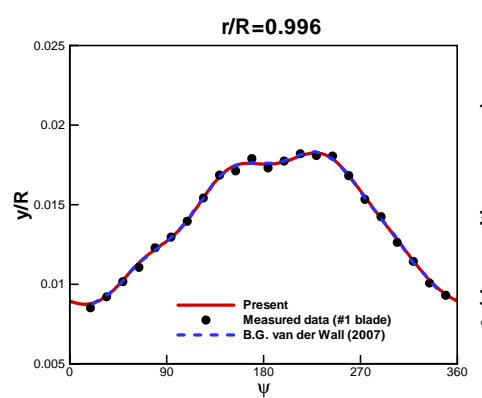

(b)lead-lag

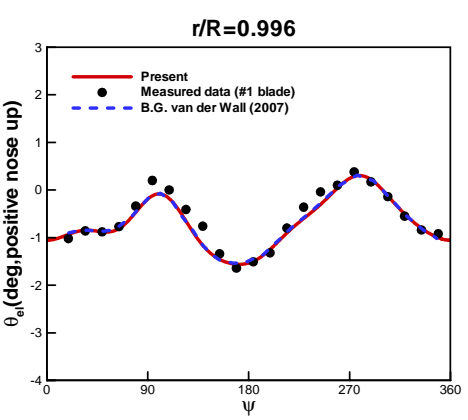

(c) torsion

Fig. 2. Measured and computed blade deflection 


\section{Dependency of airloads on grid and time step}

A grid sensitivity study was performed to verify the grid spacing effect on BVI airloads of the baseline (BL) case. Coarse and standard inner-background grids with the same size of blade grid (Table 1) were applied and blade motion from the experiment reported in Table 2 was directly used without a trim analysis for this sensitivity study. The fifth-order WENO scheme and an azimuthal increment of 0.2 degrees are used. Figure 3 shows a comparison of grid dependency for the $\mathrm{M}^{2} \mathrm{C}_{\mathrm{n}}$ at $87 \%$ span. Overall variation of the normal force coefficient in Fig. 3 (a) agrees well with experimental data and shows 3/rev harmonics. Close views around the advancing and retreating sides show most BVI peaks are well captured for both grids with the present prescribed deformation and experimental blade motion, although the control setting is not trimmed. The amplitudes of BVI peaks for the standard grid are larger than those for the coarse grid. Figure 4 shows the $\mathrm{M}^{2} \mathrm{C}_{\mathrm{m}}$ prediction at $87 \%$ span. There is a marginal difference in the $\mathrm{M}^{2} \mathrm{C}_{\mathrm{m}}$ variation between the coarse and standard grids for the present baseline case.

Another sensitivity study on the effect of azimuth angle increment was performed with increments of 1.0, 0.2, and 0.1 degrees for the coarse grid. Figures 5 and 6 display a comparison of variations of the normal force and pitching moment coefficients. The result with a 1.0 deg increment has no BVI peaks in the advancing side and shows unacceptable magnitude and locations of BVI peaks in the retreating side, whereas differences in blade loading for 0.2 and 0.1 degree increments are much smaller than those for the 1.0 degree case. Considering the BVI causes an abrupt and high-frequency airload variation, proper selection of the azimuth angle increment is imperative in capturing BVI phenomena.

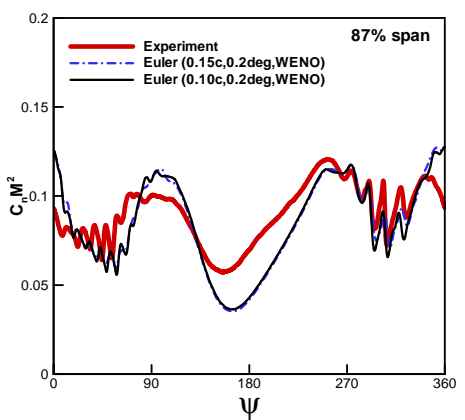

(a) whole regoin

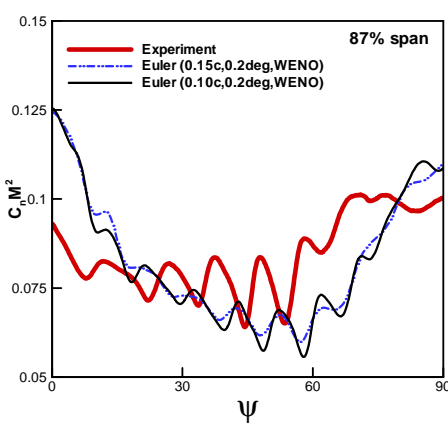

(b) advancing side

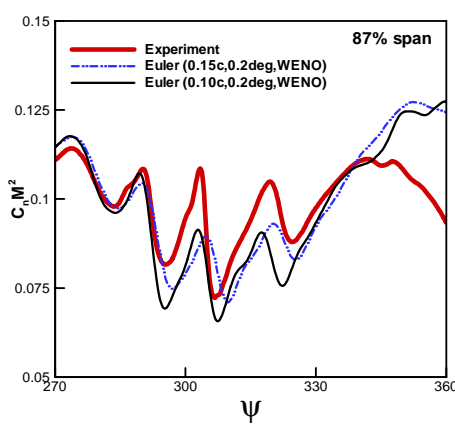

(c) etreating side

Fig. 3. $M^{2} C_{n}$ at $87 \%$ span with grid resolution

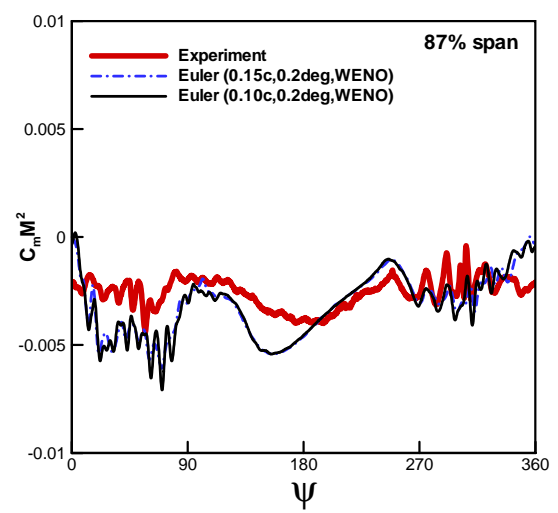

Fig. 4. Comparison of $\mathrm{M}^{2} \mathrm{C}_{\mathrm{m}}$ at $87 \%$ span with grid resolution

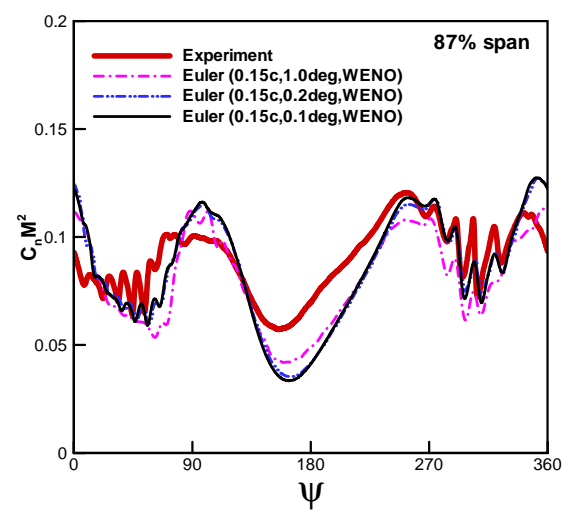

Fig. 5. Effect of azimuth angle increment for $\mathrm{M}^{2} \mathrm{C}_{\mathrm{n}}$ 
From the sensitivity study the standard grid and 0.1 degree are appropriate for the HART II baseline case, provided that a high-order spatial scheme and a 2nd-order time accurate temporal scheme are used. It is noted that for the present case dual time stepping is used to obtain second-order time accuracy. If first-order temporal accuracy is applied with the same grid, smaller azimuth increment should be applied.

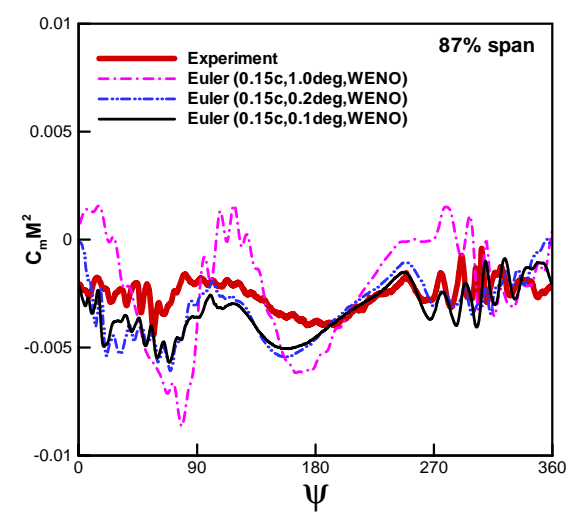

Fig. 6. Effect of azimuth angle increment for $\mathrm{M}^{2} \mathrm{C}_{\mathrm{m}}$

\section{Airload Prediction}

The measured blade first harmonics and the trim solution are presented in Table 3 . The measured harmonic values are used as initial values during the rotor trim. In the present study, the control settings were trimmed to match the measured thrust, pitching, and rolling moments in Table 2. After 4 trim cycles, thrust and moment coefficients converge to desired values.

Table 3. Thrust coefficient and blade first harmonics

\begin{tabular}{ccccc}
\hline \hline & $\mathrm{C}_{\mathrm{T}}$ & $\theta_{0}$ & $\theta 1 c$ & $\theta 1 s$ \\
\hline Experiment (BL) & 0.0044 & 3.2 & 2.0 & -1.1 \\
\hline Present & 0.0044 & 2.81 & 1.35 & -1.13 \\
\hline \hline
\end{tabular}

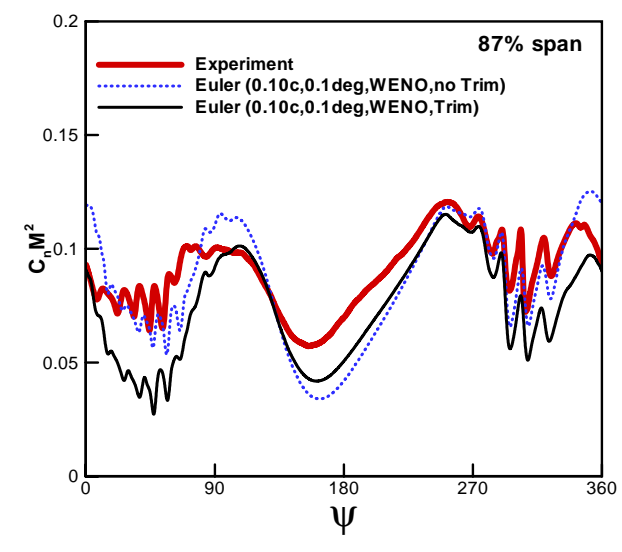

Fig. 7. Comparison of $M^{2} C_{n}$ at $87 \%$ span for Trimmed and untrimmed blade motion

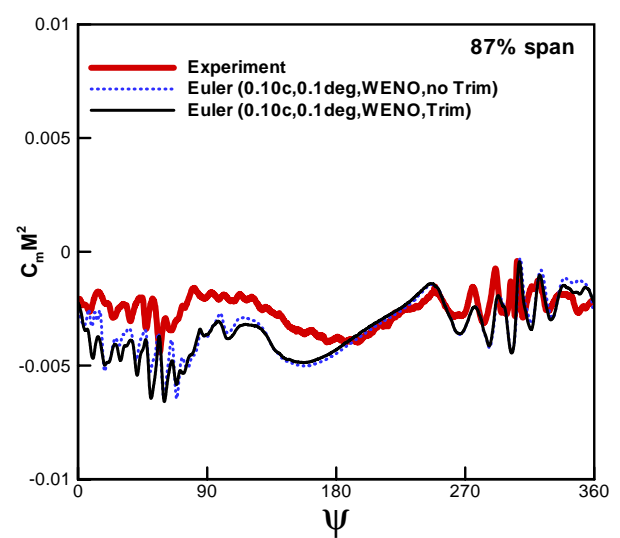

Fig. 8. Comparison of $M^{2} C_{m}$ at $87 \%$ span for Trimmed and untrimmed blade motion 


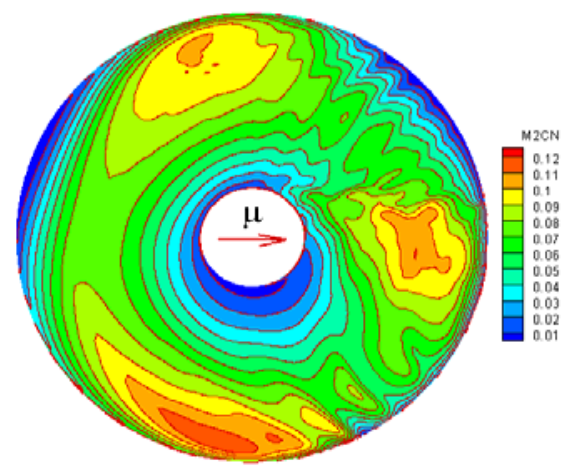

\section{Fig. 9. $\mathrm{M}^{2} \mathrm{C}_{\mathrm{n}}$ distribution for $\mathrm{BL}$ case with $0.1 \mathrm{c}$ grid and $0.1^{\circ} \quad$ azimuth angle increment}

Figures 7 and 8 display a comparison of the sectional variation of $M^{2} C_{n}$ and $M^{2} C_{m}$ for use of the measured harmonics and the trimmed harmonics with a $0.1 \mathrm{c}$ grid and a $0.1 \mathrm{deg}$. azimuth angle increment. Sectional normal forces at $87 \%$ span obtained from the trimmed solution are lower in the advancing and retreating sides than those from the measured harmonics. The difference in the force coefficient is larger than that in the moment coefficient, while the amplitude and the location of BVI peaks from the trimmed solution are similar to those for the measured harmonics. All BVI peaks are captured with reduced strength, compared to the experiment. The $\mathrm{M}^{2} \mathrm{C}_{n}$ distribution for the $\mathrm{BL}$ case is displayed in Fig. 9. The blade-vortex interaction can be seen in the $1^{\text {st }}$ and $4^{\text {th }}$ quadrants.

\section{Wake Structure Prediction}

Figure 10 illustrates the $\mathrm{Q}$ iso-surface colored by vorticity magnitude. Red color indicates high magnitude. The $\mathrm{Q}$ criterion has been used for identification of vortex structures [24]. $\mathrm{Q}$ is the balance of the vorticity magnitude and the strain rate. In the interior of a vortex, the vorticity magnitude increases and is larger than the mean strain rate. As shown in Fig. 10, tip vortices pass above the blades in the $4^{\text {th }}$ quadrant, whereas they interact with the blades in other quadrants. In the forward side around a $180^{\circ}$ azimuth angle, quite strong inboard vortices are found behind the blade. Strong root vortices, which are generated at the $22 \%$ root-cut plane, are convected downstream and interact with surrounding tip vortices, causing an interaction with the blades around the $0^{\circ}$ azimuth angle. This interaction affects blade loading around the $0^{\circ}$ azimuth angle, as shown in Fig. 9, and is discussed in $[5,13]$. Although it is not shown in this paper, a simulation with $18 \%$ root-cut plane shows that this effect of high blade loading can be reduced. The trim solution, however, is nearly the same as that with the present computation.

The tip vortex is strengthened at a position just behind the tip as the blade tip passes through from $3^{\text {rd }}$ to $1^{\text {st }}$ quadrants of the rotor disk, whereas the tip vortex is weakened in the $2^{\text {nd }}$ quadrant. This phenomenon can be seen in visualizations in references $[11,14]$. The vortices in the $2^{\text {nd }}$ quadrant do not collide with a blade, as seen in Fig. 9. In other words, there is no evidence of a vortex corruption caused from a collision with a blade. Furthermore, this is not strongly related to the strength of the circulation, which is a function of both the local tip Mach number and the pitch angle, considering that a strong tip vortex is generated in the $4^{\text {th }}$ quadrant where the effects of the tip Mach number and pitch angles are relatively lower than those in other quadrants. A reasonable explanation for the weakened strength of the tip vortex in the $2^{\text {nd }}$ quadrant was not found and this could not be explained solely by numerical dissipation.

To investigate the wake field, predicted tip vortex locations are compared with measured data identified from the DNW PIV image [25]. Selected locations lie in longitudinal cutting planes at $\mathrm{y}=1.4 \mathrm{~m}$ and $\mathrm{y}=-1.4 \mathrm{~m}$ when the reference blade is positioned at azimuth angles of 70 and 20 degrees, respectively, as shown in Fig. 11. 
Figure 11 displays a strong shear layer that merges with tip vortices in the advancing side. It should be noted that the blade moves just below the tip vortices in a nearly normal direction. The shear layer is considered to be evidence of the presence of a high speed cross-flow over the blade in the advancing side. The cross-flow in the advancing side adversely affects the strength of tip vortices. It is inferred that the weakened strength is caused not only by numerical dissipation, but also by vortex-shear layer interaction.

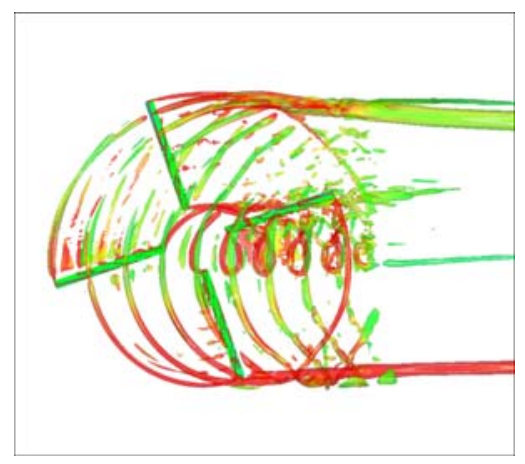

Fig. 10. $Q$ iso-surface colored by vorticity magnitude (WENO scheme, $0.1 \mathrm{c}$ )

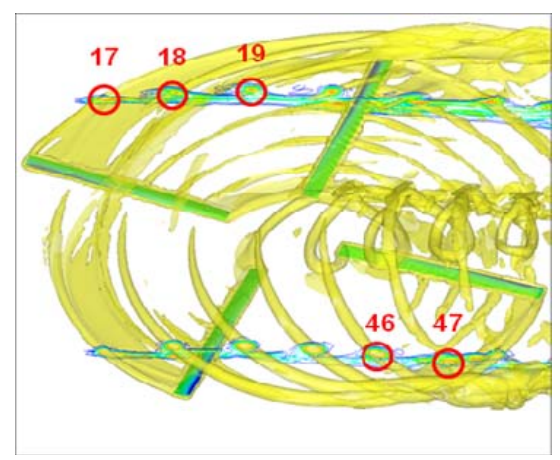

(a) $\psi=70^{\circ}$

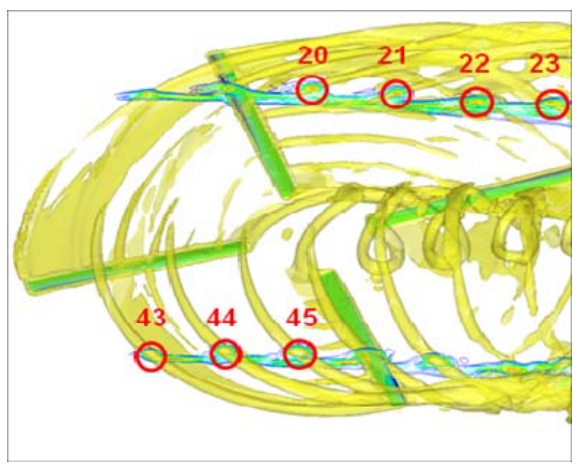

(b) $\psi=20^{*}$

Fig. 11. Vortex identification on rotor wake field: (a) the reference blade is at $70^{\circ}$, (b) at $20^{\circ}$
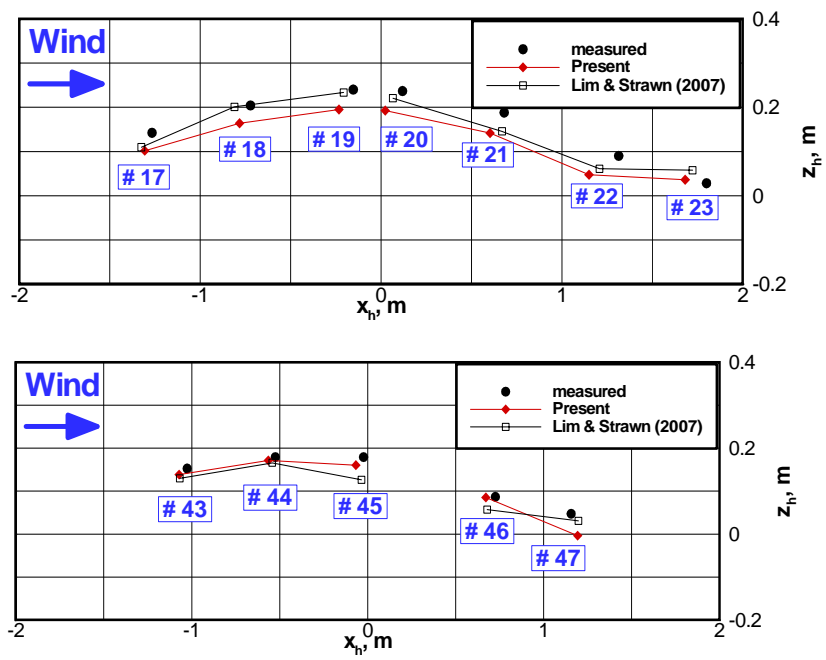

Fig. 12. Comparison of vortex locations for the HART II baseline case 
Predicted vortex locations for the HART II baseline case are compared with the measured data and OVERFLOW-II results of Lim and Strawn [11]. The OVERFLOW-II results were obtained using a fine grid system (fine near-body and fine off-body grids) with a spacing of 0.05 chords. The present prediction was performed using a standard grid system with a spacing of 0.1 chords. OVERFLOW-II results using finer grid spacing are closer to the measured data than the present prediction. The predicted tip vortex locations correlate well with the measured data and the differences are within a half chord length. The positions of vortices in the 2 nd and $3^{\text {rd }}$ quadrants are well predicted, as seen in Fig. 12. As noted in reference [11], the vortices in the $2^{\text {nd }}$ (positions 17-19) and $3^{\text {rd }}$ (positions 43-45) quadrants are younger than others. The present prediction produces nearly similar locations from a CFD/CSD coupled analysis with a standard (snb/sob) grid system, which has a similar resolution to the present grid system (not shown here). This comparison shows that the present CFD analysis with the prescribed deformation can predict BVI airloads and wake fields accurately for the HART II rotor blade.

\section{Conclusion}

KFLOW results of HART II rotor airload and wake prediction using the measured deformation are presented. The analytical expression given by van der Wall, as a prescribed deformation, is used to describe smooth variations of deformation and moving velocities for the CFD simulation. A sensitivity study on numerical parameters shows that the azimuth angle increment is a crucial parameter for accurate prediction of BVI airloads, provided that a 2nd-order accurate time stepping scheme is applied. The present approach using the prescribed deformation predicts all BVI peaks in both the advancing and retreating sides. The present standard grid with a grid spacing of 0.1 chords provides promising resolution of vortices and the wake field for the present BL case, despite having insufficient grid resolution. The predicted vortex locations correlate well with the measured data and the OVERFLOW prediction. The weakened strength of tip vortices in the $2^{\text {nd }}$ quadrant can be explained with the vortex location and the shear layer caused from a cross-flow over the blade. Finer grid systems of course should be used to resolve the wake field inside the vortex core radius. Detailed analysis of the wake structure and the shear-layer interaction with vortices is also needed.

\section{Acknowledgement}

This work was supported by the Korea Foundation for International Cooperation of Science \& Technology (KICOS) through a grant provided by the Korean Ministry of Education, Science \& Technology(MEST) in 2009 (No. K20601000001) and partially supported by the KARI under the KHP Dual-Use Component Development Program funded by the MOCIE. The authors express special thanks to the HART II project team for giving us the opportunity to use their database.

\section{References}

1. Yu, Y. H., Gmelin, B., Heller, H., Philippe, J.J., Mercker, E., and Preisser, J. S., 1994, "HHC Aeroacoustics Rotor Test at the DNW - The Joint German/French/US HART Project," 20th European Rotorcraft Forum, Amsterdam, Netherlands.

2. Yu, Y. H., Tung, C., van der Wall, B. G., Pausder, H., Burley, C., Brooks, T., Beaumier, P., Delrieux, Y., Mercker, E., and Pengel, K., 2002, "The HART-II Test: Rotor Wakes and Aeroacoustics with Higher-Harmonic Pitch Control (HHC) Inputs - The Joint German/French/Dutch/US Project," American Helicopter Society 58th Annual Forum, Montreal, Canada.

3. Van der Wall, B. G., Junker, B., Burley, C. L., Brooks, T., Yu, Y. H., Tung, C., Raffel, M., Richard, H., Wagner, W., Mercker, E., Pengel, K., Holthusen, H., Beaumier, P., and Prieur, 
J., 2002, "The HART-II Test in the LLF of the DNW-a Major Step towards Rotor Wake Understanding," 28th European Rotorcraft Forum, Bristol, England.

4. Bailly, J., Delrieux, Y., and Beaumier, P., 2004, "HART-II: Elemental Analysis and Validation of ONERA Methodology for the Prediction of Blade-Vortex Interaction", 30th European Rotorcraft Forum, Marseilles, France.

5. Lim, J. W., and van der Wall, B. G., 2005, "Investigation of the Effect of a Multiple Trailer Free Wake Model for Descending Flights", AHS 61st Annual Forum, Grapevine, TX.

6. Van der Wall, B. G., Yin. J., 2007, "DLR's S4 Rotor Code Validation With HART II Data: The Baseline Case”, American Helicopter Society Specialists' Conference, Seoul, Korea.

7. Dietz, M., Kramer, E., and Wagner, S., 2006, "Tip Vortex Conservation on a Main Rotor in Slow Descent Flight Using Vortex-Adapted Chimera Grids”, AIAA paper 2006-3478.

8. Brown, R. E. and Line, A. 2005, "Efficient High-Resolution Wake Modeling using the Vorticity Transport Model”, AIAA Journal, Vol. 43, No. 7, pp. 1434-1443.

9. Kelly, M. E., and Brown, R. E., 2005, "The effect of Blade Aerodynamic Modeling on the Prediction of High-Frequency Rotor Airloads”, AHS $65^{\text {th }}$ Annual Forum, Grapevine, TX.

10. Renaud, T., Perez, G., Benoit, C., and Peron, S., 2008, "Blade-Vortex Interaction Capture by CFD," 34th European Rotorcraft Forum, Liverpool, England.

11. Lim, J. W., and Strawn, R. C., 2007, "Prediction of HART II Rotor BVI Loading and Wake System Using CFD/CSD Loose Coupling”, AIAA-2007-1281, $45^{\text {th }}$ AIAA Aerospace Sciences Meeting and Exhibit, Reno, NV.

12. Yang, C., and Aoyama, T., 2008, "Effect of Computation Parameters on BVI Noise Prediction Using HART II Motion Data”, 34th European Rotorcraft Forum, Liverpool, England.

13. Boyd, D. D, 2005, "HART-II Acoustic Predictions using a Coupled CFD/CSD Method", AHS $65^{\text {th }}$ Annual Forum, Grapevine, TX.

14. Van der Wall, B. G., 2007, "Mode identification and data synthesis of HART II blade deflection data”, Institute Report, IB-111-2007/28, ftp://HART-II@ftp.dlr.de.

15. Kim, J. W., Park, S. H., and Yu, Y. H., 2009, "Euler and Navier-Stokes Simulations of Helicopter Rotor Blade in Forward Flight Using an Overlapped Grid Solver", AIAA2009-4268, $19^{\text {th }}$ AIAA CFD Conference, San Antonio, TX.

16. Jameson, A., 1991, "Time Dependent Calculations Using Multigrid with Applications to Unsteady Flows Past Airfoils and Wings," AIAA Paper 91-1596.

17. Park, S. H., and Kwon, J. H., 2004, "Implementation of $k-\omega$ Turbulence Models in an Implicit Multigrid Method.”, AIAA Journal, Vol. 42, No. 7, pp. 1348-1357.

18. Jiang, G. S., and Shu, C. W., 1999, "Efficient Implementation of Weighted ENO Schemes", Journal of Computational Physics, Vol.126, No. 1, pp. 202-228.

19. Thomas, P.D., and Lombard, C.K., 1979, "Geometric Conservation Law and its Application to Flow Computations on Moving Grids", AIAA Journal, Vol. 17, pp. 1030-1037.

20. Kim, E., Park, S. H., and Kwon, J. H.,2006, "Parallel Performance Assessment of Moving Body Overset Grid Application on PC Cluster", Parallel Computational Fluid Dynamics - Parallel Computing and Its Applications, edited by J. H. Kwon et al., Busan, Korea, pp. 59-66.

21. Cho, K. W., Kwon J. H., and Lee, S., 1998, "Development of a Fully Systemized Chimera Methodology for Steady/Unsteady Problems", Journal of Aircraft, Vol. 36, No. 6, pp. 973-980.

22. Schneider, O., van der Wall, B. G., and Pengel, K., 2003, "HART II Blade Motion Measured by Stereo Pattern Recognition (SPR)", Proceedings of the American Helicopter Society 59th Annual Forum, Phoenix, Arizona.

23. Pengel, K., Mueller, R. H. G., and van der Wall, B. G., 2002, "Stereo Pattern Recognition - the Technique for Reliable Rotor Blade Deformation and Twist Measurement", Proceedings of the American Helicopter Society International Meeting on Advanced Rotorcraft Technology and Life Saving Activities (Heli Japan), Tochigi, Utsunomiya, Japan.

24. Dubief, Y., and Delcayre, F., 2000, "On Coherent-Vortex Identification in Turbulence", Journal of Turbulence, Vol.1, Article No.11.

25. Van der Wall, B. G., and Richard, H., 2006, "Analysis Methodology for 3C-PIV Data of Rotary Wing Vortices”, Experiments in Fluids, Vol. 40, No. 5, pp. 798-812. 